\title{
石墨烯一铁酸铋纳米晶复合材料的制备及其催化性能研究
}

\author{
李 铁 ${ }^{1}$, 李 玥 $^{1}$, 王颖异 ${ }^{2}$, 张 珽 $^{1}$ \\ (1. 中国科学院 苏州纳米技术与纳米仿生研究所, 创新实验室, 苏州 $215123 ; 2$. 西交利物浦大学 理学院, 健康与 \\ 环境科学系，苏州 215123)
}

摘 要: $\mathrm{BiFeO}_{3}(\mathrm{BFO})$ 是一种新型可回收光响应催化剂, 但较高的光生电子/空穴对复合率和较低的量子产率限制了 其实际应用。本研究通过水热法制备出还原氧化石墨烯-BFO(RGO-BFO)纳米晶复合材料, 表征与测试结果表明, 相比于 $\mathrm{BFO}$ 颗粒，复合材料的禁带宽度 $E_{\mathrm{g}}$ 为 $2.0 \mathrm{eV}$ ，降低约 $10 \%$; $40 \mathrm{~min}$ 对亚甲基蓝吸附-催化效率接近 $100 \%$, 远 高于 $\mathrm{BFO}$ 颗粒 $(28 \%)$, 这主要由于复合体系中光生电子/空穴对复合率更低。通过本征磁性回收并重复利用 6 次后, 复合材料仍保持 $89.1 \%$ 催化效率, 表现出优异催化性能。

关 键 词：铁酸铋; 纳米晶; 石墨烯; 催化降解

中图分类号: O643 文献标志码: A

\section{Preparation and Catalytic Properties of Graphene-Bismuth Ferrite Nanocrystal Nanocomposite}

\begin{abstract}
LI Tie $^{1}$, LI Yue ${ }^{1}$, WANG Yingyi ${ }^{2}$, ZHANG Ting ${ }^{1}$
(1. International Laboratory for Adaptive Bio-nanotechnology (i-LAB), Suzhou Institute of Nano-Tech and Nano-Bionics, Chinese Academy of Sciences, Suzhou 215123, China; 2. Department of Health and Environmental Science, School of Science, Xi'an Jiao-tong Liverpool University, Suzhou 215123, China)
\end{abstract}

\begin{abstract}
BiFeO}_{3}(\mathrm{BFO})$ is a novel recyclable photocatalyst which benefits from its appropriate theoretical band gap and room temperature ferromagnetism, yet the relatively high recombination rate of photogenerated electron-hole pairs and low quantum yield limit its practical catalytic performance. Here, a composite material of reduced graphene oxide and $\mathrm{BiFeO}_{3}$ nanocrystals (RGO-BFO) was successfully prepared via a hydrothermal self-assembly process with about 10wt \% RGO loaded $85 \mathrm{wt} \%$ BFO nanocrystalline $(\sim 10 \mathrm{~nm})$. Specific surface area of the composite is about 5 times of that of pure BFO particles. Furthermore, this nanocomposite demonstrates the enhanced ultraviolet absorption behavior as well as its intrinsic visible light absorption, with an energy gap $\left(E_{\mathrm{g}}\right)$ of $2.0 \mathrm{eV}$, which is about $10 \%$ lower than BFO particles $(2.3 \mathrm{eV})$. As a result, a significantly higher catalytic efficiency of nearly $100 \%$ of the RGO-BFO was obtained as compared to the $25 \%$ value of BFO after a 40 -min process of adsorption and photodegradation for methylene blue. The improved performances may be attributed to the additional photogenerated electron-hole pairs and lower recombination ratio, which can be proved by the result of photoelectric response. As an intrinsically ferromagnetic material, it could be easily recycled from the solvent
\end{abstract}

收稿日期：2020-10-19; 收到修改稿日期：2021-01-27；网络出版日期：2021-03-01

基金项目：国家重点研发计划(2017YFA0701101, 2020YFB2008501); 国家自然科学基金(62071462, 51702354); 中国科学院 青年促进会(2020320); 江苏省基础研究计划面上项目(BK20201195); 苏州市重点产业技术创新项目 (SYG202029)

National Key R\&D Program of China (2017YFA0701101, 2020YFB2008501); National Natural Science Foundation of China (62071462, 51702354); Youth Promotion Association of Chinese Academy of Sciences (2020320); Foundation Research Project of Jiangsu Province (BK20201195); Suzhou Key Industrial Technology Innovation Project (SYG202029)

作者简介：李 铁(1984-), 男，副研究员.E-mail: tli2014@sinano.ac.cn LI Tie(1984-), male, associate professor. E-mail: tli2014@sinano.ac.cn 
system, and its repetitive catalytic efficiency maintained $89.1 \%$ after 6 cycles, showing a good catalytic stability.

Key words: bismuth ferrite; nanocrystals; graphene; catalytic degradation

多铁性材料是同时具有铁电性、铁磁性、铁弹 性中两种或三种性能的一类多功能材料 ${ }^{[1]}$ 。作为一 种典型的单相多铁性磁电材料, $\mathrm{BiFeO}_{3}(\mathrm{BFO})$ 在室 温下兼具两种结构有序, 即铁电有序和反铁磁有序, 是少数几种在室温下兼有铁电性和寄生弱铁磁性的 多铁性材料之一 ${ }^{[2-3]}$, 因此受到研究者长期关注 ${ }^{[4]}$ 。 另外, BFO 具有合适的理论禁带宽度 $(2.18 \sim 2.50 \mathrm{eV})$ 和常温铁磁性, 可以作为一种可回收的新型光响应 催化材料用于光分解水或降解有机污染物, 在催化 领域展现出良好的应用前景 ${ }^{[5-6]}$ 。然而, 较高的光生 电子/空穴对复合率和较低的量子产率极大地限制了 BFO 在实际使用过程中的催化效能 ${ }^{[7]}$ 。研究表明 ${ }^{[8]}$, 通过提升对目标降解物的吸附性能, 并降低材料内 部光生载流子/空穴对的重组速率, 可以有效提高 BFO 的光催化效率。通常将 BFO 与具有大比表面 积、良好电子/离子传导能力的基底材料进行复合, 通过控制 BFO 颗粒尺寸来增加催化活性位点的暴 露数量, 并结合基底所引入的电子或空穴捕获中心 来抑制载流子重组, 已成为提高 $\mathrm{BFO}$ 光催化效能的 有效手段 ${ }^{[9]}$ 。

石墨烯具有大的比表面积、稳定的化学性质和 良好的电子或空穴传递能力, 作为复合基底材料可 使光催化剂内部产生的电子和空穴快速分离, 降低 它们的重组率 ${ }^{[10]}$, 因此, 研究者将其作为催化剂载 体来提升复合材料的光催化性能 ${ }^{[11]}$ 。目前, 金属 $(\mathrm{Cu} 、 \mathrm{Pt} 、 \mathrm{Pd} 、 \mathrm{Au} 、 \mathrm{Ag})$ 或金属氧化物 $\left(\mathrm{TiO}_{2} 、 \mathrm{Fe}_{3} \mathrm{O}_{4}\right.$ 、 $\left.\mathrm{SnO}_{2} 、 \mathrm{MnO}_{2} 、 \mathrm{Co}_{3} \mathrm{O}_{4} 、 \mathrm{ZnO}\right)$ 与石墨烯的复合已有相 关研究报道 ${ }^{[12-15]}$ 。但是, 有关调控石墨烯一铁酸铋 (RGO-BFO)复合材料的晶体结构及其与催化性能之 间关系的研究报道较少, 且提升该复合体系光催化 性能的机制也有待深入探索。本研究采用一步水热 法制备的 RGO-BFO 纳米晶复合材料 $(<10 \mathrm{~nm})$, 与 纯 BFO 颗粒 $(30 \mathrm{~nm} / 50 \mathrm{~nm})$ 材料相比, 在磁学和光学 性能, 以及降解有机染料的效率上都有所增强, 在 工业催化方面展现出良好的应用前景。

\section{1 实验方法}

\section{1 原材料与试剂}

可膨胀石墨 8099200( 180 $\mu \mathrm{m})$ 由青岛百川石墨 有限公司提供 $\left(1000{ }^{\circ} \mathrm{C}\right.$ 时可膨胀原体积的 1000 倍左
右)。高锰酸钾 $\left(\mathrm{KMnO}_{4}\right)$ 、浓硫酸、双氧水 $\left(30 \% \mathrm{H}_{2} \mathrm{O}_{2}\right)$ 、 硝酸铋 $\left(\mathrm{Bi}\left(\mathrm{NO}_{3}\right)_{3} \cdot 5 \mathrm{H}_{2} \mathrm{O}\right)$ 、硝酸铁 $\left(\mathrm{Fe}\left(\mathrm{NO}_{3}\right)_{3} \cdot 9 \mathrm{H}_{2} \mathrm{O}\right)$ 、 氢氧化钾 $(\mathrm{KOH})$ 、抗坏血酸 $(\mathrm{Vc})$ 和亚甲基蓝( $(\mathrm{MB})$ 等 由上海国药集团提供, 均为分析纯, 直接使用未做 进一步处理。

\section{2 测试与表征}

红外光谱(FTIR)分析: NEXUS 670 美国 Nicolet 公司; 拉曼光谱(Raman)分析: Dilor LABRAM-1B 型; 热失重(TGA)分析仪: Netzsch TG 209F1; X 射线 粉末衍射(XRD): Rigaku D/max- $\gamma \mathrm{B}, \lambda=0.154056 \mathrm{~nm}$; 透射电镜(TEM)及选区电子衍射(SAED)分析: JEOL 2010F (JEOL Ltd., Japan); 扫描电子显微镜(SEM)及 $X$ 射线能量谱(EDX)分析: Philips XL30 FEG FE-SEM, $25 \mathrm{kV} ; \mathrm{X}$ 射线光电子能谱(XPS)分析: XR $5 \mathrm{VG}$ (UK) 使用 Mg X-ray 源; 原子力显微镜(AFM) 分析: Veeco Dimension 3100; 磁性 (VSM) 测试: MPMS-XL-7, Quantum Design Inc., USA(5 K 和 $300 \mathrm{~K}$ ); 紫外-可 见光谱(UV-Vis): APADA UV-1800PC, 扫描范围为 $720 \sim 250 \mathrm{~nm}$ ，扫描速率为 $2 \mathrm{~nm} / \mathrm{min}$ 。

\subsection{Hummer's 法制备氧化石墨烯}

采用改进 Hummer's 法制备氧化石墨烯(GO), 具体过程如下: 首先, 在冰浴条件下将石墨 $(5 \mathrm{~g})$ 加 入到 $98 \%$ 浓 $\mathrm{H}_{2} \mathrm{SO}_{4}(115 \mathrm{~mL})$ 中, 边搅拌边加入 $\mathrm{KMnO}_{4}(15 \mathrm{~g})$, 严格控制加入试剂速度和反应温度 (不超过 $20{ }^{\circ} \mathrm{C}$ ); 然后, 将混合物加热到 $35{ }^{\circ} \mathrm{C}$, 反应 $30 \mathrm{~min}$ 后缓慢加入 $230 \mathrm{~mL}$ 去离子水, 随后迅速升 温至 $98{ }^{\circ} \mathrm{C}$ 并反应 $15 \mathrm{~min}$; 继续加入 $700 \mathrm{~mL}$ 去离子 水与 $50 \mathrm{~mL} \mathrm{H}_{2} \mathrm{O}_{2}(30 \%)$ 混合溶液, $2 \mathrm{~h}$ 后停止反应, 将 产物离心洗涤至中性后在 $65{ }^{\circ} \mathrm{C}$ 下真空下干燥备用。

\subsection{RGO-BFO 纳米晶复合材料的制备}

采用一步水热法制备 RGO-BFO 复合材料, 制 备过程为: (1)将所制备的 $\mathrm{GO}(10 \mathrm{mg})$ 和 $\mathrm{Vc}(1 \mathrm{mg})$ 加 入到 $10 \mathrm{~mL}$ 去离子水中, 超声分散 $1 \mathrm{~h}$ 至 $\mathrm{GO}$ 完全 溶解, 形成组分 $\mathrm{A}$; (2) 将 $2 \mathrm{mmol}$ 等化学计量比的 $\mathrm{Bi}\left(\mathrm{NO}_{3}\right)_{3} \cdot 5 \mathrm{H}_{2} \mathrm{O}$ 和 $\mathrm{Fe}\left(\mathrm{NO}_{3}\right)_{3} \cdot 9 \mathrm{H}_{2} \mathrm{O}$ 分别溶解在 $\mathrm{KOH}$ 溶液中 $(8 \mathrm{~mol} / \mathrm{L}, 50 \mathrm{~mL})$, 得到组分 $\mathrm{B}$; (3)混合组分 A、B 并磁力搅拌 $30 \mathrm{~min}$ 后, 将混合溶液倒入装有 聚四氟乙烯内祄水热釜中, 在 $180{ }^{\circ} \mathrm{C}$ 温度下反应 $6 \mathrm{~h}$; (4)用去离子水和乙醇反复洗涤反应产物数次至洗 涤液为中性, 然后置于 $60{ }^{\circ} \mathrm{C}$ 真空烘箱中干燥 $12 \mathrm{~h}$ 即得 RGO-BFO 复合材料。 


\section{5 光降解 $\mathrm{MB}$ 实验}

将 $100 \mathrm{mg}$ 催化剂(RGO-BFO)加入到 $100 \mathrm{~mL}$ $\left(10^{-5} \mathrm{~mol} / \mathrm{L}\right) \mathrm{MB}$ 溶液中, 搅拌 $30 \mathrm{~min}$ 后达到吸附平 衡。然后, 加入 $\mathrm{H}_{2} \mathrm{O}_{2}(10 \mu \mathrm{L})$ 在紫外光 $(1 \mathrm{~kW})$ 光照条 件下磁力摚拌溶液, 并每隔一定时间取样 $3 \mathrm{~mL}$, 过 滤后用紫外分光计检测溶液中的 MB 吸收峰, 分析 其溶度值。

\section{2 结果与讨论}

RGO-BFO 纳米晶复合材料的制备过程如图 1 所示, 其机理可归纳为 ${ }^{[10,16]}$ : 首先, 由于 $\mathrm{GO}$ 表面存 在大量带负电荷含氧官能团，如 $-\mathrm{COOH} 、-\mathrm{COH}$ 、 $-\mathrm{OH}$ 等, 在库仑引力相互作用下使带正电荷的金属 阳离子 $\mathrm{Bi}^{3+}$ 和 $\mathrm{Fe}^{3+}$ 吸附在其表面; 其次, 在碱性溶 液中, $\mathrm{Bi}^{3+}$ 和 $\mathrm{Fe}^{3+}$ 与氨水电离出的 $\mathrm{OH}^{-}$相结合, 在 $\mathrm{GO}$ 表面生成 $\mathrm{Bi}(\mathrm{OH})_{3}$ 和 $\mathrm{Fe}(\mathrm{OH})_{3}$ 沉淀。在水热升温 过程中, GO 在碱性溶液中逐渐被 $\mathrm{Vc}$ 还原为 $\mathrm{RGO}$; 同时，在高温高压水热条件下, $\mathrm{Bi}(\mathrm{OH})_{3}$ 与 $\mathrm{Fe}(\mathrm{OH})_{3}$ 之间发生矿化反应，在 RGO 表面成核并结晶生成 $\mathrm{BFO}$ 。由于还原生成的 RGO 在此反应条件下相互之 间存在着较强的范德华力相互作用，有强烈聚集、 团聚或重新石墨化的趋势, 从而在 $\mathrm{RGO}$ 二维片层 之间产生一定限域作用，使得 $\mathrm{BFO}$ 晶核仅生长为纳 米晶结构; 同时，由于表面负载 BFO 纳米晶之间的 静电排斥作用，阻止了 RGO 的重新聚集或团聚，最 终制备得到 RGO-BFO 纳米晶复合材料。

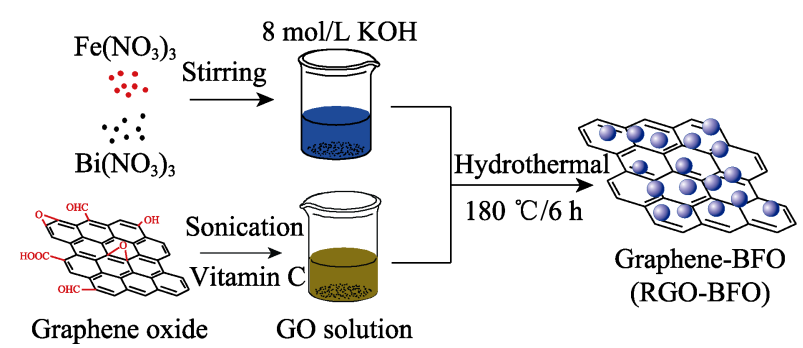

图 1 RGO-BFO 制备流程示意图

Fig. 1 Preparation process illustration of the RGO-BFO nanocrystal nanocomposite

\section{1 形貌与 BET 的表征分析}

利用 SEM/EDX、TEM/SEAD 及 AFM 等表征 RGO-BFO 纳米晶复合材料的形貌并分析其成分, 结果如图 2 所示。从图 2(a)中可知, 大量尺寸均一 的 $\mathrm{BFO}$ 极细纳米粒子均匀地负载在几乎透明的 $\mathrm{RGO}$ 之上; 同时, $\mathrm{GO}$ 被还原成 $\mathrm{RGO}$ 后表面产生皱 褶现象(箭头标示处), 能更好地增强祄底与负载物 $\mathrm{BFO}$ 纳米晶之间界面的接触和相互作用，从而提高 复合材料的离子/电子扩散能力 ${ }^{[16]}$ 。从图 2(b)的成分 分析结果可知, RGO-BFO 中 $\mathrm{Fe}$ 与 $\mathrm{Bi}$ 两元素含量之 比约为 $1: 1$, 说明复合体系分子结构与理论 $\mathrm{BFO}$ 结 构式相同。另外，从图 2(c)中 TEM 结果可见，尺 寸均一的 $\mathrm{BFO}$ 纳米晶均匀分布在 $\mathrm{RGO}$ 祄底之上, 这与 SEM 观测结果相一致。样品(图 2(c)标红圈区 域)的 SEAD 图案呈现出环形的多晶衍射环特征 (图 2(d)), 说明产物中 BFO 呈纳米尺寸极细晶粒形 态。进一步利用 AFM 确定 BFO 纳米晶尺寸约为
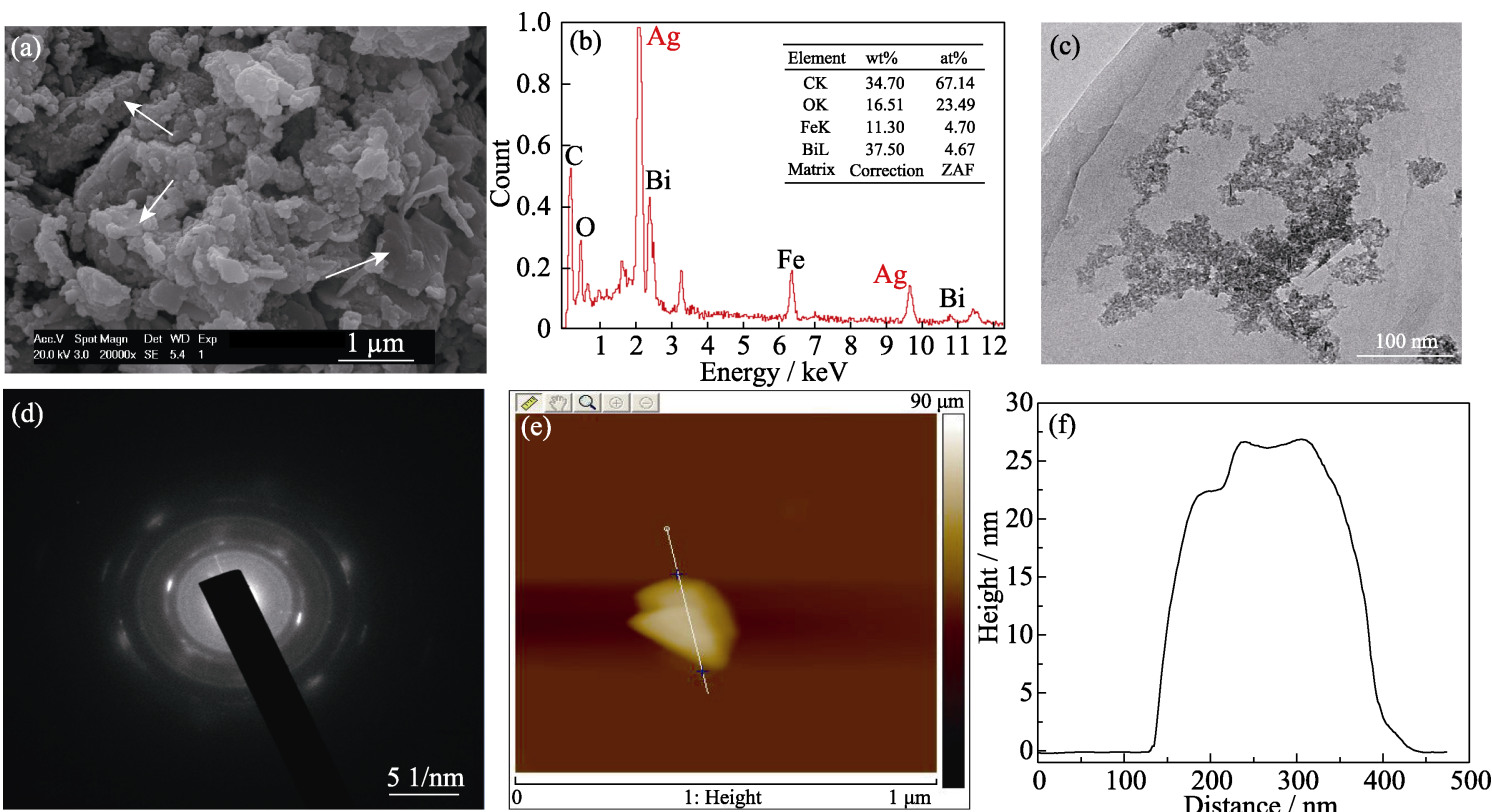

图 2 RGO-BFO 纳米晶复合材料的形貌和成分分析结果

Fig. 2 Morphology and composition characterizations of RGO-BFO nanocrystal nanocomposite (a) SEM/EDX; (c-d) TEM/SEAD and (e-f) AFM 
$10 \mathrm{~nm}$ (2 3 个 BFO 颗粒叠加尺寸约为 $20 \mathrm{~nm}$ ), 如 图 2(e f) 所示, 这与根据 XRD 衍射峰计算得到的晶 粒尺寸相一致。上述结果表明, 产物为 RGO-BFO 纳米晶复合体系。

纳米晶复合体系具有大的比表面积、良好的界 面离子/电子扩散能力等特征, 对与表/界面性质相 关的理化性能有重要影响, 例如电化学性能、催化 性能及吸附性能等。复合体系及对比 BFO 颗粒样品 的 BET 分析结果如表 1 所示, 由大比表面积 RGO 片层及 $\mathrm{BFO}$ 纳米晶体结构所组成 RGO-BFO 纳米晶 复合样品的 BET 比表面积为 $23.124 \mathrm{~m}^{2} / \mathrm{g}$, 约为 $\mathrm{BFO}$ 颗粒 $\left(30 \mathrm{~nm}\right.$ )样品面积的 5 倍 $\left(4.763 \mathrm{~m}^{2} / \mathrm{g}\right)$, 这有利于 提升复合材料催化或吸附等性能。另外, 从结果也 可知, 与易发生团聚造成其孔直径和体积较小的 BFO 颗粒样品相比, RGO-BFO 具有更小的孔体积 和直径, 这是由于大比表面积 RGO 负载 BFO 纳米 晶后，可使其均匀分散且更不易发生团聚，也有利 于吸附和提升催化性能。

\section{2 结构与组成的表征分析}

图 3(a)为 GO 及 RGO-BFO 纳米晶复合材料的 $\mathrm{XRD}$ 图谱。从图可知, 复合样品衍射峰 $\left(2 \theta=22.5^{\circ}\right.$ 、 $31.8^{\circ} 、 32.1^{\circ} 、 39.5^{\circ} 、 45.8^{\circ} 、 51.4^{\circ} 、 57.0^{\circ} 、 57.1^{\circ}$ 及
65.2ํำ均与标准 BFO 图谱(JCPDS 86-1518)相符合, 属扭曲三方晶系钙钛矿结构 ${ }^{[17]}$, 说明实验制备了纯 $\mathrm{BFO}$ 单相材料。根据谢乐公式计算得到 BFO 晶粒 尺寸约 $12 \mathrm{~nm}$, 与 $\mathrm{AFM}$ 的分析结果基本一致。另外, 没有检测到 GO 所对应 $2 \theta=18.5^{\circ}$ 处(002)晶面衍射 峰 $^{[18]}$, 说明 GO 被完全还原为 $\mathrm{RGO}$, 且在高温高压 条件下未发生团聚、聚集或重新石墨化, 这是由于 吸附在 RGO 表面成晶核并生长的 $\mathrm{BFO}$ 纳米晶能阻 止 RGO 单片层重新聚集, 使 RGO 团聚或石墨化趋 势降低。图 3(b)为 BFO、RGO-BFO、RGO 以及 $\mathrm{GO}$ 的红外光谱图。在 $\mathrm{BFO}$ 曲线中位于 500 和 $650 \mathrm{~cm}^{-1}$ 低频处的吸收峰, 分别对应 BFO 晶体结构中 $\mathrm{Bi}-\mathrm{O}$ 四面体和八面体位置 $\mathrm{Fe}-\mathrm{O}$ 键伸缩振动峰 ${ }^{[19]}$ 。在 $\mathrm{RGO}-\mathrm{BFO}$ 谱图中能观测到与 $\mathrm{BFO}$ 同波段位置振动 吸收峰，也说明复合体系中只存在纯 BFO 相，这与 $\mathrm{XRD}$ 分析结果相一致。相比于 $\mathrm{GO}$ 的 FI-TR 谱图, 复 合体系中没有出现包括 $1700 \mathrm{~cm}^{-1}$ 处 $\mathrm{C}=\mathrm{O}$ 键、 1250 和 $1235 \mathrm{~cm}^{-1}$ 处 $\mathrm{C}-\mathrm{O}$ 键以及 $750 \sim 650 \mathrm{~cm}^{-1}$ 处 $-\mathrm{OH}$ 键 等在内绝大部分含氧基团吸收峰，只出现了位于 $1620 \mathrm{~cm}^{-1}$ 处 $-\mathrm{C}=\mathrm{C}$ 双键及 $1100 \mathrm{~cm}^{-1}$ 处 $-\mathrm{C}-\mathrm{C}$ 单键的 $\mathrm{C}$ 骨架结构的吸收峰, 证实了反应过程中 $\mathrm{GO}$ 能够 被完全还原为 RGO, 并负载 BFO 纳米晶制备得到

表 1 RGO-BFO 复合体系与 BFO 的比表面积对比表

Table 1 Comparision of specific surface area between RGO-BFO composite and BFO

\begin{tabular}{lccccc}
\hline \multicolumn{1}{c}{ Sample } & $S_{\mathrm{BET}} /\left(\mathrm{m}^{2} \cdot \mathrm{g}^{-1}\right)$ & $S_{\text {Langmuir }} /\left(\mathrm{m}^{2} \cdot \mathrm{g}^{-1}\right)$ & $S_{\text {T-method }} /\left(\mathrm{m}^{2} \cdot \mathrm{g}^{-1}\right)$ & $V_{\text {pore } \text { HK method }} /\left(\mathrm{cm}^{3} \cdot \mathrm{g}^{-1}\right)$ & $D_{\text {pore, HK method }} / \mathrm{nm}$ \\
\hline RGO-BFO & 23.124 & 39.536 & 23.1240 & 0.00651 & 0.3675 \\
BFO & 4.763 & 7.237 & 1.8463 & 0.15020 & 1.9020 \\
\hline
\end{tabular}
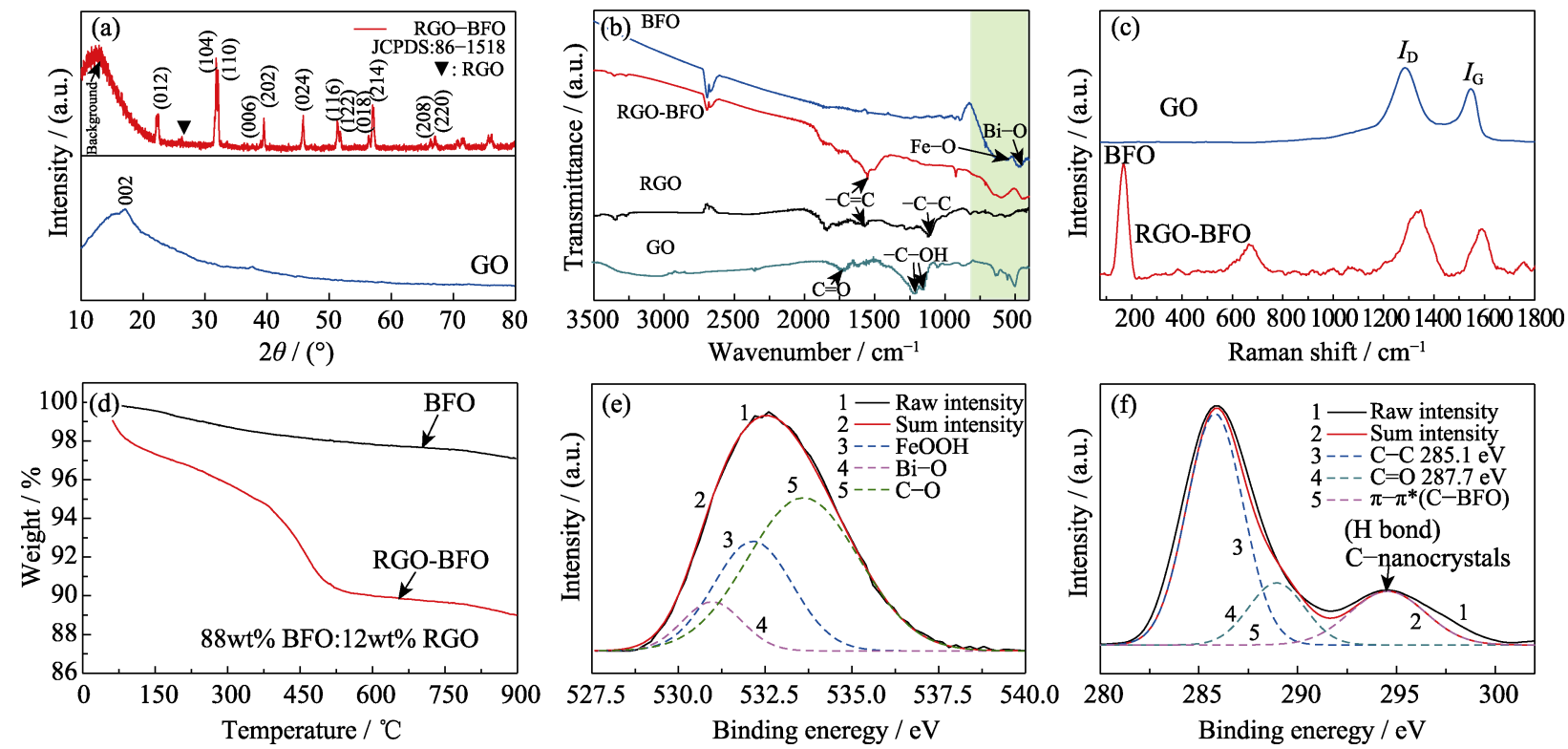

图 3 RGO-BFO 纳米晶复合材料的结构和组成

Fig. 3 Structure and composition analysis of RGO-BFO nanocrystal nanocomposite (a) XRD patterns; (b) FT-IR spectra; (c) Raman spectra; (d) TG curves; (e-f) XPS peaks 
纳米复合材料。拉曼光谱对于石墨电子结构的改变 响应性较强, 可用来表征复合材料的电子结构 ${ }^{[20]}$ 。

图 3(c)是原料 GO 与产物 RGO-BFO 复合体系的 Raman 光谱图, 从图中可知, 复合材料体系中位于 $1300 \mathrm{~cm}^{-1}$ 处石墨缺陷 D 峰与位于 $1580 \mathrm{~cm}^{-1}$ 处 $\mathrm{E}_{2 \mathrm{~g}}$ 模式 $\mathrm{G}$ 峰的强度比 $I_{\mathrm{D}} / I_{\mathrm{G}}$ 大于 $\mathrm{GO}$ 相, 说明 $\mathrm{GO}$ 被完 全还原为 $\mathrm{RGO}^{[21]}$ 。另外, 复合材料 $\mathrm{RGO}$ 的 $\mathrm{D}$ 峰变 强变宽, 进一步说明体系中 $\mathrm{RGO}$ 和 $\mathrm{BFO}$ 纳米晶之 间存在强的电荷传输关联效应，表面负载的 BFO 纳 米晶在阻止 $\mathrm{GO}$ 高温高压下重新石墨化趋势的同时, 可以使 RGO 中产生更多无定型缺陷结构, 这有利 于提升复合材料对活性物质的吸附能力，增加复合 体系反应活性位点 ${ }^{[22]}$ 。另外, 复合材料中位于约 $600 \mathrm{~cm}^{-1}$ 处四面体 $\mathrm{AO}_{4}$ 结构 $\mathrm{A}_{1}$ 振动模式和 $190 \mathrm{~cm}^{-1}$ 处八面体 $\mathrm{BO}_{6}$ 结构金属离子振动模式, 与纯 $\mathrm{BFO}$ 单 相材料八面体结构 $\mathrm{M}-\mathrm{O}$ 键中 $\mathrm{O}$ 原子对称和反对称 振动模式相吻合 ${ }^{[23]}$, 也说明复合体系中只有纯 $\mathrm{BFO}$ 单相，这与 XRD 及 FT-IR 分析结果相一致。用热失 重分析仪测试复合材料的热稳定性能和负载量, 结 果如图 3(d)所示, 可以看出, 在空气气氛下纯 BFO 颗粒在 $900{ }^{\circ} \mathrm{C}$ 以下失重不到 $3 \mathrm{wt} \%$ ，说明其具有良 好高温稳定性。对于 RGO-BFO 复合材料, $150{ }^{\circ} \mathrm{C}$ 以 下的失重(约 $3 \mathrm{wt} \%$ ) 主要是由游离水和结晶水蒸发 造成的; $150{ }^{\circ} \mathrm{C}$ 到 $900{ }^{\circ} \mathrm{C}$ 失重约 $10 \mathrm{wt} \%$ ，其主要失重 温度在 $450{ }^{\circ} \mathrm{C}$ 左右, 这是典型碳材料(RGO)主体结 构分解和燃烧温度 ${ }^{[24]}$ 。由此计算可得，复合材料是 由约 $10 \mathrm{wt} \%$ 的 $\mathrm{RGO}$ 基底负载了约 $85 \mathrm{wt} \%$ 的 $\mathrm{BFO}$ 纳
米晶组成, 纳米级的负载量高, 有利于吸附和催化。

用 XPS 表征 RGO-BFO 纳米晶复合材料中元素 的基本状态, 结果如图 3(e f) 所示。在 $\mathrm{O} 1 \mathrm{~s}$ 谱图中 位于 533.6、532.2 及 $530.9 \mathrm{eV}$ 处的能级峰, 分别对 应于 $\mathrm{FeOOH} 、 \mathrm{Bi}-\mathrm{O}$ 及 $\mathrm{C}-\mathrm{O}$ 价键，前两者均属于 $\mathrm{BFO}$ 结构中 $\mathrm{O} 1 \mathrm{~s}$ 对应 $\mathrm{O}^{2-}$ 离子能级峰, 说明复合材料中 纯 $\mathrm{BFO}$ 相并不含诸如 $\mathrm{FeO} 、 \mathrm{Fe}_{3} \mathrm{O}_{4} 、 \mathrm{Fe}_{2} \mathrm{O}_{3} 、 \mathrm{Bi}_{2} \mathrm{Fe}_{4} \mathrm{O}_{9}$ 和 $\mathrm{Bi}_{25} \mathrm{FeO}_{40}$ 等杂相, 这与 XRD 及 Raman 分析结果 相一致。另外, 在 C1s 谱图中位于 294.7、287.7 和 $285.1 \mathrm{eV}$ 处能级峰, 分别对应于 $\pi-\pi$ 键、 $\mathrm{C}=\mathrm{O}$ 键和 $\mathrm{C}-\mathrm{C}$ 键。比较峰积分面积可知, 产物中 $\mathrm{C}$ 原子主要 处于 $\mathrm{sp}^{2}$ 杂化态, 说明在反应中原料 $\mathrm{GO}$ 片层中含氧 基团(如 $\mathrm{HO}-\mathrm{C}=\mathrm{O} 、 \mathrm{C}=\mathrm{O}=\mathrm{C}$ 和 $\mathrm{C}-\mathrm{OH}$ 等)大部分已被 还原去除，这证实了 FT-IR 的分析结果。值得注意 的是, 还原后在 $\mathrm{C} 1 \mathrm{~s}$ 谱图中位于 $294.7 \mathrm{eV}$ 处出现了 代表芳香族共轭体系的 $\pi-\pi$ 键能级峰, 说明复合材 料中 RGO 处于高度分散状态，这是由于在复合材 料表面负载的大量 $\mathrm{BFO}$ 纳米晶阻止了 $\mathrm{RGO}$ 在高温 高压反应中重新聚集或石墨化 ${ }^{[25]}$ 。综上所述, 采用 水热法可制备出具有 BFO 单晶体结构的 RGO-BFO 纳米晶复合材料。

\section{3 磁学、光学及催化性能分析}

BFO 材料具有磁性, 通过 RGO-BFO 复合体系 及 $\mathrm{BFO}$ 的磁化曲线 $(M-H)$ (图 4(a))研究 RGO 负载 BFO 纳米晶对复合材料磁性的影响。从结果可知, 在 $300 \mathrm{~K}$ 时复合材料磁化强度达到 $275 \mathrm{~A} / \mathrm{m}$, 约为 $\mathrm{BFO}$ 的 1.6 倍 $(176 \mathrm{~A} / \mathrm{m})$, 说明纳米晶结构的 $\mathrm{BFO}$
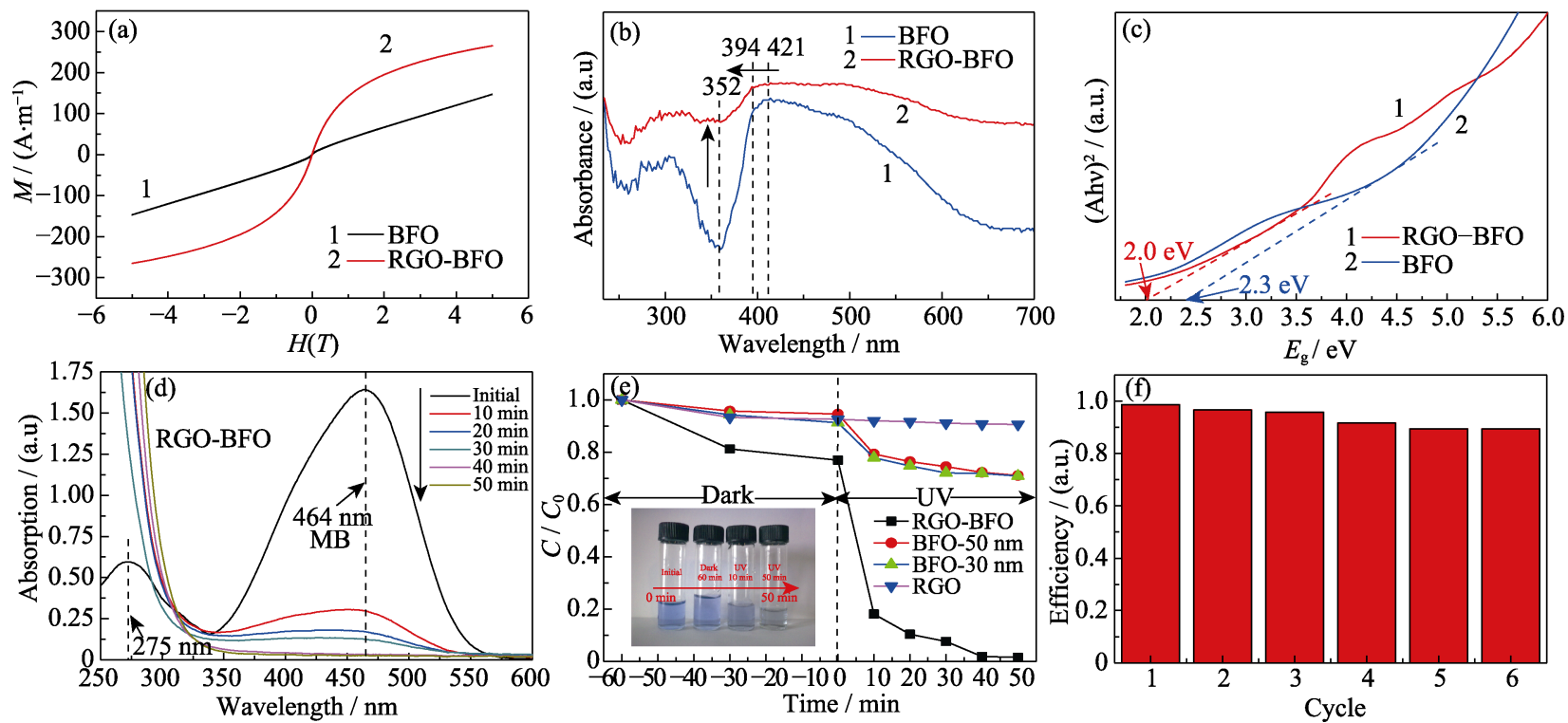

图 4 RGO-BFO 纳米晶复合材料性质及紫外光催化降解 MB 性能图

Fig. 4 Physical properties and photocatalytic MB degradation performances of RGO-BFO nanocrystal nanocomposite (a) $M-H$ magnetic curve; (b-c) UV-Vis absorption spectra and calculated result of optical band gap; (d) Absorption spectra of MB solution under various time nodes; (e) Degradation effects of various controlled samples; (f) Cyclic performance of the composite 
加入石墨烯对材料磁学性能影响较大, 这可能是由 于纳米晶结构本征磁性与块体不同, 加入石墨烯同 时也增强了材料的本征磁性。而具备磁学性能的 RGO-BFO 复合材料易于吸附, 利用磁性可将其从 催化环境中分离出来并重复循环使用, 从而节约成 本。图 4(b, c) 是 RGO-BFO 复合材料的紫外-可见光 (UV-Vis) 吸收光谱及光禁带图。从图 4(b) 可知, BFO 只在 $421 \mathrm{~nm}$ 波长处有强吸收峰, 这是由于结构中价 带向导带产生的电子跃迁 $\left(\mathrm{O}_{2 \mathrm{p}}{ }^{2-} \rightarrow \mathrm{Fe}_{3 \mathrm{~d}}{ }^{3+}\right)^{[26]}$; 而 RGO-BFO 复合材料不仅可见光吸收波段移至 $394 \mathrm{~nm}$, 而且在紫外光波段(352 nm) 也产生了较强 吸收, 说明加入 RGO 能有效提高复合材料光谱吸 收强度并拓宽吸收范围, 即不仅提升了 RGO-BFO 复合材料的 BFO 本征可见光催化性能, 而且增强了 紫外光催化能力, 这可能与石墨烯材料本身良好的 光电传导性能及全波段吸收能量相关。另外, 通过 UV-Vis 光谱可计算出 RGO-BFO 材料的禁带宽度。 禁带宽度 $E_{\mathrm{g}}$ 可以通过式(1)计算得到:

$$
(A h v)^{2}=B\left(h v-E_{\mathrm{g}}\right)
$$

其中, $h v$ 是光子能量 $E_{\text {photon }}(\mathrm{eV}), A$ 代表吸收系数, $B$ 是与材料性质相关的常数。以 $(A h v)^{2}$ 为纵坐标、

$E_{\text {photon }}$ 为横坐标做光禁带图, 将切线部分外推至零 点, 与横坐标的交点即为材料的 $E_{\mathrm{g}}$ 值, 结果如图 4(c) 所示。采用相同方法制备 $\mathrm{BFO}$ 材料的 $E_{\mathrm{g}}$ 值为 $2.3 \mathrm{eV}$, 而 RGO-BFO 复合材料的 $E_{\mathrm{g}}$ 值则为 $2.0 \mathrm{eV}$, 光禁带 值减小约 $10 \%$, 这进一步说明加入 $\mathrm{RGO}$ 改变了复 合材料体系的光学性能。禁带宽度的减小有助于增 强光电子的传输, 并阻止电子空穴重排, 从而有效 地增强催化剂的催化活性。

基于以上分析, 本研究采用紫外光催化降解染 料分子 MB 模型分析 RGO-BFO 纳米晶复合材料的 光催化性能。先将材料放在暗室中搅拌足够长时间 以确保达到吸附平衡之后再进行光催化降解。在紫 外光降解过程中检测 $\mathrm{MB}$ 在 $464 \mathrm{~nm}$ 处吸光度来分 析不同时间材料的降解能力和效率, 结果如图 4(d) 所示。从图 4(d)中可以看出, 随着紫外光降解时间 的延长, 在 $464 \mathrm{~nm}$ 处的 $\mathrm{MB}$ 吸收峰强度快速下降, 表明复合材料催化剂具有高效的催化活性。随着降 解后 $\mathrm{MB}$ 溶液溶度的降低, 复合材料在 $464 \mathrm{~nm}$ 处的 吸收峰朝着短波方向发生蓝移, 并且 $275 \mathrm{~nm}$ 处的吸 收峰消失, 这与 $\mathrm{MB}$ 降解后共轭结构的破坏有关 ${ }^{[27]}$ 。 进一步通过建立 $C / C_{0}\left(C_{0}\right.$ 表示 $\mathrm{MB}$ 溶液初始溶度, $C$ 表示降解 $t$ 时刻后溶液溶度) 随时间变化的规律, 分 析纳米晶复合材料和不同尺寸 $\mathrm{BFO}$ 纳米颗粒 $(\sim 50 \mathrm{~nm} / \sim 30 \mathrm{~nm})$ 的紫外光降解效率, 结果如图 4(e)
所示。从平衡吸附结果可知, RGO-BFO 纳米晶复合 体系的平衡吸附率最高 (23.1\%), 分别比 RGO (7.4\%)、50 nm (5.4\%)及 $30 \mathrm{~nm}(8.7 \%)$ 的 BFO 颗粒吸 附率高出 $15.7 \%$ 、 $17.7 \%$ 及 $14.4 \%$; 且比 $\mathrm{RGO}$ 与 $50 \mathrm{~nm} / 30 \mathrm{~nm}$ BFO 颗粒吸附率之和还分别高出 $10.3 \%$ 与 $7.0 \%$, 说明复合体系中 $\mathrm{RGO}$ 与 $\mathrm{BFO}$ 纳米 晶并不是简单功能相加, 而是相互之间产生了协同 吸附作用。另外, BFO 纳米颗粒的吸附效率随颗粒 尺寸的减小而略微增大，这是由于尺度越小，颗粒 表面能越高, 更有利于吸附。从紫外光降解结果可 知, RGO 紫外光降解 MB 能力相比于对比样可以忽 略不计, 效率低于 $3 \%$, 总效率低于 $8 \%$; 而不同尺 寸 BFO 颗粒紫外光催化效率总体相近(约 23\%), 总 效率约为 $28 \%$ 。相比而言, RGO-BFO 纳米晶复合材 料 $10 \mathrm{~min}$ 紫外光降解 $\mathrm{MB}$ 效率就达到约 $60 \%$, 40 min 降解 MB 效率就达到约 $80 \%$, 说明具有优良 的紫外光降解性能。另外, 加上平衡吸附效率, RGO-BFO 复合材料整体降解效率接近 $100 \%$, 远高 于 $\mathrm{RGO}$ 和 $\mathrm{BFO}$ 纳米颗粒总体效率。

磁学性能结果表明磁性有利于 RGO-BFO 复合 材料从催化环境中分离出来并可重复利用。如图 4(f) 所示 RGO-BFO 纳米晶复合材料循环使用 6 次的实 验结果，催化效率分别达到首次催化活性的 $98.7 \%$ 、 $96.7 \% 、 95.8 \% 、 91.7 \% 、 89.5 \%$ 及 $89.1 \%$ ，依然保持 较高的催化活性, 说明 RGO-BFO 纳米晶复合体系 是一种性能优良且循环使用效率高的光催化剂。

\section{4 紫外光催化机制分析}

RGO-BFO 纳米晶复合材料具有较高紫外光催 化性能是由于石墨烯具有芳香结构, 染料分子能通 过 $\pi-\pi$ 键相互作用吸附到 RGO 表面; 同时 RGO 表 面还原产生的缺陷可形成活性位点, 从而在其周围 吸附更多的染料分子形成更高的催化平衡溶度, 因 而相互协同作用增强了复合材料光催化效率。利用 紫外光生电流的测试结果对比分析了 $\mathrm{BFO}$ 和复合 体系紫外光光电化学特性, 结果如图 5(a)所示。从 图中可以看出, 在紫外光照射下 RGO-BFO 复合材 料的光电流急速增大, 随后达到一个稳定值; 当停 止照射时, 光电流又迅速下降, 整个过程可以重复。 同时, RGO-BFO 复合材料的光电流强度是 BFO 的 6 倍, 而光电流强度与电子-空穴对的数量及存活时 间直接相关 ${ }^{[28]}$, 紫外光生电流越强说明电子-空穴 数量越多、可存活时间越长, 表明 RGO-BFO 复合 体系在紫外光照射下产生的电子-空穴对数量和存 活时间远超过 BFO 材料, 而电子-空穴对又正相关 于催化降解性能。这是由于石墨烯具有类似大 $\pi$ 键 

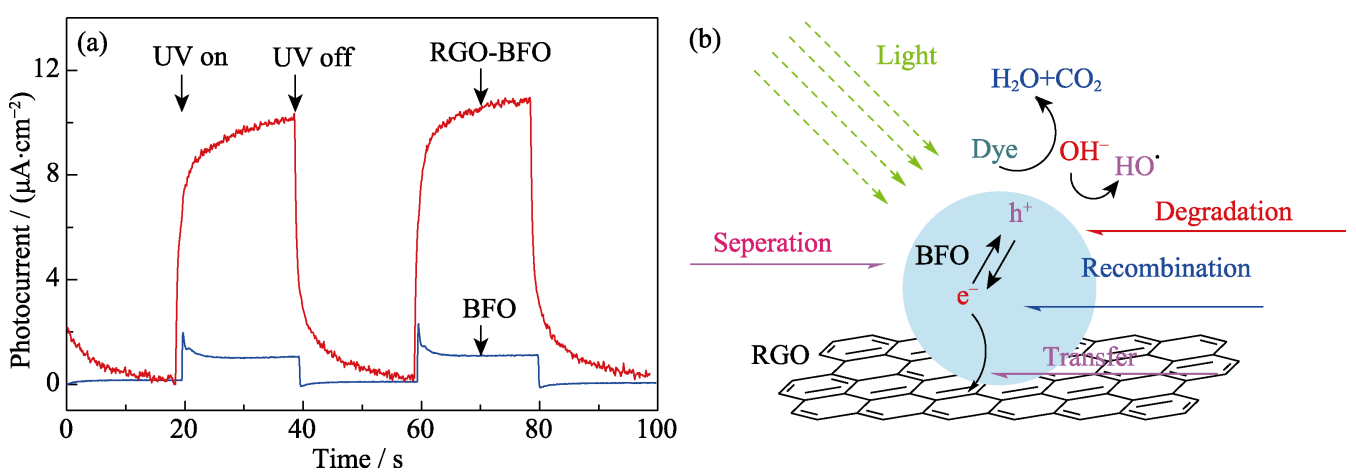

图 5 RGO-BFO 纳米晶复合材料光电响应机制

Fig. 5 Photoelectric response mechanism of RGO-BFO nanocrystal nanocomposite (a) Photogenerated currents of the controlled sample; (b) Degradation mechanism of the nanocrystal composite

的芳香结构，可使 BFO 表面产生的光激发电子转移 到石墨烯上，从而延长电子-空穴对的存活时间，增 大光电流强度; 同时 RGO 的紫外吸收能力和 BFO 的高负载率也会在某种程度上提高光电流的强度。 因此，以上分析可归纳为(如图 5(b))：当紫外光照射 到复合材料时，体系中的 BFO 产生大量电子-空穴 对, 一方面电子 ( $\mathrm{e}^{-}$) 通过 $\mathrm{RGO}$ 祄底材料转移 (Transferring)并与之苯环结构发生吸电子共轭现象, 而被分离的空穴 $\left(\mathrm{h}^{+}\right)$则与 $\mathrm{H}_{2} \mathrm{O}_{2}$ 电离 $\mathrm{OH}^{-}$共同作用下, 将染料分子 (Dye) 降解生成二氧化碳 $\left(\mathrm{CO}_{2}\right)$ 与水 $\left(\mathrm{H}_{2} \mathrm{O}\right)$, 从而实现对染料分子的降解(Degradation)。 在此过程中, 生成 $\mathrm{OH}^{\prime}$ 维持整个体系的电荷平衡, 且在紫外光停止照射后能与电子 $\left(\mathrm{e}^{-}\right)$重新复合 (Recombination), 使电子-空穴对再次达到平衡, 以 便循环使用。另外, 由于对电子 $\left(\mathrm{e}^{-}\right)$的转移, 引入石 墨烯可以降低电子-空穴对复合率，使其存活时间 更长，这也有利于提升光催化效率 ${ }^{[29-30]}$ 。

\section{3 结论}

本研究以 $\mathrm{Bi}\left(\mathrm{NO}_{3}\right)_{3} 、 \mathrm{Fe}\left(\mathrm{NO}_{3}\right)_{3}$ 和 $\mathrm{GO}$ 为前驱体, 在碱性条件下以 $\mathrm{Vc}$ 为还原剂, 通过一步水热法合 成出 RGO-BFO 纳米晶 $(<10 \mathrm{~nm})$ 复合材料。反应过 程中, $\mathrm{RGO}$ 二维片层之间的限域作用使得 $\mathrm{BFO}$ 仅能 生长成纳米晶结构, 同时负载 $\mathrm{BFO}$ 纳米晶又能阻止 $\mathrm{RGO}$ 在高温高压下重新团聚或石墨化。由于 $\mathrm{BFO}$ 纳米晶与 RGO 祄底之间相互协同作用, RGO-BFO 纳米晶复合体系不仅原有 $\mathrm{BFO}$ 本征可见光吸收特 性得到提升, 而且其紫外光吸收特性得到较大增 强。复合材料体系展现出远优于 BFO 纳米 $(>10 \mathrm{~nm})$ 颗粒材料的光催化降解性能, $40 \mathrm{~min}$ 对亚甲基蓝吸 附-催化效率接近 $100 \%$ ，这主要得益于复合体系在 紫外光下能产生更多的光生电子-空穴对, 且电子-
空穴对复合率下降、存活时间更长。另外，复合材 料体系的磁性使其能从溶剂中回收并重复使用，且 具有良好的循环利用效率。

\section{参考文献:}

[1] 卢鹏, 胡雪利, 赖昕, 等. 铁酸铋的制备及其在光催化领域的研 究进展. 应用化工, 2018, 6(47): 1270-1273.

[2] ILIEV M N, LITVINCHUK A P, LEE H G, et al. Raman spectroscopy of $\mathrm{SrRuO}_{3}$ near the paramagnetic-to-ferromagnetic phase transition. Physical Review B, 1999, 59(1): 364-369.

[3] ALI S, HUMAYUN M, PI W, et al. Fabrication of $\mathrm{BiFeO}_{3}$-g- $\mathrm{C}_{3} \mathrm{~N}_{4}-\mathrm{WO}_{3}$ Z-scheme heterojunction as highly efficient visible-light photocatalyst for water reduction and 2,4-dichlorophenol degradation: insight mechanism. Journal of Hazardous Materials, 2020, 397: 122708 .

[4] LIU J, NIU M, WANG L, et al. Effect of tuning A/B substitutions on multiferroic characteristics of $\mathrm{BiFeO}_{3}$-based ternary system ceramics. Journal of Magnetism and Magnetic Materials, 2020, 510: 166928

[5] ZHAO R, MA N, SONG K, et al. Boosting photocurrent via heating $\mathrm{BiFeO}_{3}$ materials for enhanced self-powered UV photodetectors. Advanced Functional Materials, 2020, 30: 1906232.

[6] HAUMONT R, KREISEL J, BOUVIER P, et al. Phonon anomalies and the ferroelectric phase transition in multiferroic $\mathrm{BiFeO}_{3}$. Physical Review B, 2006, 73(13): 132101.

[7] ZOU C, LIU S, SHEN Z, et al. Efficient removal of ammonia with a novel graphene-supported $\mathrm{BiFeO}_{3}$ as a reusable photocatalyst under visible light. Chinese Journal Catalysis, 2017, 38(1): 20-28.

[8] SI Y, XIA Y, SHANG S, et al. Enhanced visible light driven photocatalytic behavior of $\mathrm{BiFeO}_{3} /$ reduced graphene oxide composites. Nanomaterials, 2018, 8: 526 .

[9] GHOSH S, DASGUPTA S, SEN A, et al. Low-temperature synthesis of nanosized bismuth ferrite by soft chemical route. Journal of the American Ceramic Society, 2005, 88(5): 1349-1952.

[10] KHOMCHENKO V A, SHVARTSMAN V V, BORISOV P, et al. Effect of Gd substitution on the crystal structure and multiferroic properties of $\mathrm{BiFeO}_{3}$. Acta Materialia, 2009, 57(17): 5137-5145.

[11] KIANI M, RIZWAN S, IRFAN S. Facile synthesis of a $\mathrm{BiFeO}_{3} /$ nitrogen-doped graphene nanocomposite system with enhanced photocatalytic activity. Journal of Physics and Chemistry Solids, 2018, 121: 8-16.

[12] LIN X, SAMIA A C S. Synthesis, assembly and physical properties of magnetic nanoparticles. Journal of Magnetism and Magnetic 
Materials, 2006, 305(1): 100-109.

[13] ZHOU Q, LIN Y, ZHANG $\mathrm{K}$, et al. Reduced graphene oxide $/ \mathrm{BiFeO}_{3}$ nanohybrids-based signal-on photoelectrochemical sensing system for prostate-specific antigen detection coupling with magnetic microfluidic device. Biosensors and Bioelectronics, 2018, 101: 146-152.

[14] LATTUADA M, HATTON T A. Preparation and controlled self-assembly of Janus magnetic nanoparticles. Journal of the American Chemical Society, 2007, 129(42): 12878-12889.

[15] CHENZ, WANG Y, ZHENG D, et al. Polarization tunable and enhanced photovoltaic properties in tetragonal-like $\mathrm{BiFeO}_{3}$ epitaxial films with graphene top electrode. Journal of Alloys and Compounds, 2019, 811: 152013.

[16] 胡玉林, 李永进, 谢燕春, 等. 掺 $\mathrm{Ni}$ 铁酸铋纳米粉的制备及光 催化性能. 材料导报(B), 2020, 34(9): 18009-18013.

[17] SEUNG W L, CHUL S K. Growth of multiferroics $\mathrm{BiFeO}_{3}$ thin films by Sol-Gel method. Journal of Magnetism and Magnetic Materials, 2006, 304(12): 772-774.

[18] IRFAN S, LIANG G, LI F, et al. Effect of graphene oxide nano-sheets on structural, morphological and photocatalytic activity ofBiFeO ${ }_{3}$-based nanostructures. Nanomaterials, 2019, 9(9): 1337.

[19] PARK T J, MAO Y B, STANISLAUS S. Synthesis and characterization of multiferroic $\mathrm{BiFeO}_{3}$ nanotubes. Chemical Communications, 2004, 12(23): 2708-2709.

[20] SHEN J, HU Y, QIN C, et al. Layer-by-layer self-assembly of multiwalled carbon nanotube polyelectrolytes prepared by in situ radical polymerization. Langmuir, 2008, 24(8): 3993-3997.

[21] KIANIM, KIANI A B, KHAN S A, et al. Facile synthesis of Gd and $\mathrm{Sn}$ co-doped $\mathrm{BiFeO}_{3}$ supported on nitrogen doped graphene for enhanced photocatalytic activity. Journal of Physics and Chemistry of Solids, 2019, 130: 222-229.

[22] CARUSO F, LICHTENFELD H, DONATH E, et al. Investigation of electrostatic interactions in polyelectrolyte multilayer films: binding of anionic fluorescent probes to layers assembled onto colloids. Macromolecules, 1999, 32(7): 2317-2328.

[23] LIU T, XU Y B, FENG S S, et al. A facile route to the synthesis of $\mathrm{BiFeO}_{3}$ at low temperature. Journal of the American Ceramic Society, 2011, 94(9): 3060-3063.

[24] ZHANG S X, WANG L, GAO Z S. Ferromagnetism in sub-micron scale $\mathrm{BiFeO}_{3}$. Materials Letters, 2011, 65(21/22): 3309-3312.

[25] REN Y, NAN F, YOU L, et al. Enhanced photoelectrochemical performance in reducedgraphene oxide $/ \mathrm{BiFeO}_{3}$ heterostructures. Small, 2017, 13(16): 1603457.

[26] MKHOYAN K A, CONTRYMAN A W, SILCOX J, et al. Atomic and electronic structure of graphene-oxide. Nano Letters, 2009, 9(3): 1058-1063

[27] MOITRA D, GHOSH B K, CHANDEL M, et al. Synthesis of a $\mathrm{BiFeO}_{3}$ nanowire-reduced graphene oxide based magnetically separable nanocatalyst and its versatile catalytic activity towards multiple organic reactions. RSC Advances, 2016, 6: 97941.

[28] EDA G, CHHOWALLA M. Graphene-based composite thin films for electronics. Nano Letters, 2009, 9(2): 814-818.

[29] BLAKE P, BRIMICOMBE P D, NAIR R R, et al. Graphene-based liquid crystal device. Nano Letters, 2008, 8(6): 1704-1708.

[30] LI Z, SHEN Y, GUAN Y, et al. Bandgap engineering and enhanced interface coupling of graphene- $\mathrm{BiFeO}_{3}$ nanocomposites as efficient photocatalysts under visible light. Journal of Materials Chemistry A, 2014, 2: 1967-1973. 